\title{
Regulation mechanism of biomolecule interaction behaviors on the superlubricity of hydrophilic polymer coatings
}

\author{
Caixia ZHANG ${ }^{1,2}$, Junmin CHEN ${ }^{1}$, Mengmeng LIU ${ }^{2}$, Yuhong LIU $^{3}$, Zhifeng LIU $^{1,2}$, Hongyan CHU ${ }^{1,2, *}$, \\ Qiang CHENG ${ }^{2}$, Jianhua WANG ${ }^{1,2}$ \\ ${ }^{1}$ Institute of Advanced Manufacturing and Intelligent Technology, Beijing University of Technology, Beijing 100124, China \\ ${ }^{2}$ Beijing Key Laboratory of Advanced Manufacturing Technology, Beijing University of Technology, Beijing 100124, China \\ ${ }^{3}$ State Key Laboratory of Tribology, Tsinghua University, Beijing 100084, China \\ Received: 18 April 2020 / Revised: 09 June 2020 / Accepted: 10 August 2020 \\ (C) The author(s) 2020.
}

\begin{abstract}
Hydrophilic polymer coatings can improve the surface characteristics of artificial implants. However, because they are used in vivo, they inevitably come into contact with biomolecules that affect their interfacial tribological properties. In this paper, the friction behaviors of poly(vinylphosphonic acid) (PVPA)-modified Ti6Al4V and polytetrafluorethylene balls were analyzed using albumin, globulin, aggrecan, and hyaluronic acid as lubricants. The interaction properties and dynamic adsorption characteristics of the biomolecules and PVPA molecules were explored by a quartz crystal microbalance to identify the cause of the friction difference. It was found that protein molecules disturbed the superlubricity of the PVPA-phosphate-buffered saline system because of the formation of a stable adsorption film, which replaced the interfacial characteristics of the PVPA coating. Polysaccharides, with their excellent hydration properties and polymer structure, had an unstable dynamic interaction or zero adsorption with PVPA molecules, and hardly changed the superlubricity of the PVPA and phosphate-buffered-saline system. The influence mechanism of the specific friction of proteins and polysaccharides was analyzed. Interactions were observed among different biomolecules. Polysaccharides can potentially reduce protein adsorption. The result of the synergistic regulation of the friction coefficient for PVPA-modified Ti6Al4V is approximately 0.017. The results of this study will provide a theoretical basis for the use of polymer coatings in vivo.
\end{abstract}

Keywords: superlubricity; hydrophilic polymer; protein; polysaccharide

\section{Introduction}

Artificial implants can help restore the functional movement/mobility of people suffering from joint disease or recovering from an accident. Current biomaterials research on artificial implants has focused on the improvement of wear resistance, such as metallic, polymeric, ceramic, and novel biomimetic materials and potential combinations of materials to extend the life of the implant [1]. Titanium alloy is one of the most widely used materials in artificial joints because of its excellent biocompatibility and good mechanical strength [2-4]. However, its applications are limited by their low wear resistance [5]. Attempts have been made to improve the tribological properties of titanium alloys. In recent years, the application of surface texture [6], surface coatings, and modifications have realized superlubricity under certain conditions [7]. The superlubricity [8,9] of natural joints in biological systems is an important target that needs to be achieved when designing artificial implants.

* Corresponding author: Hongyan CHU, E-mail: chuhongyan@bjut.edu.cn 
Surface modification of titanium alloys has been widely used to enhance its tribological properties [10-15]. Inspired by the ultralow friction of biological systems, surfaces have been modified with hydrophilic polymer brushes, which can effectively mimic the characteristics of natural cartilage. This method has attracted considerable attention [16]. 2-Methacryloyloxyethyl phosphorylcholine has been widely researched [17-20] because of its unique amphoteric characteristics. In biological tissues, the main reasons for the low friction are the highly hydrated phosphocholine groups, the strong van der Waals attraction between tails, and the suppressed interpenetration between two tribo-pairs [21]. Poly (ethylene glycol) (PEG) is a potential candidate for surface modification of titanium alloys. The chain length, grafting density, and branching structure of PEG have been investigated [22-25]. Sulfonic polymers also show good tribological properties [26]. When the phosphate group is hydrated, superlubricity occurs in the phosphonic polymer coating, which is not affected by different loads and speeds [27, 28].

Considering the application of artificial implants in vivo, the modified surfaces inevitably come into contact with biomolecules in body fluids in addition to water molecules [29]. To the best of our knowledge, the inflammatory response is a major response related to the interface of artificial implants. For example, nonspecific protein adsorption is thought to trigger collagen layer formation, which then sends a signal to the immune system. Therefore, the interaction between biomolecules and implant surfaces has attracted the attention of numerous researchers. The hydration layer formed on the polymer surface is vital for protein adsorption. Hydrophilic polymers, such as PEG, polyacrylamide, and polyzwitterions, have been found to effectively resist the attachment of protein and bacteria $[18,30]$. Furthermore, the cationic and anionic polymer layers exhibit significantly different characteristics compared with neutral hydrophilic polymer or zwitterionic polyelectrolyte surfaces, even though they all show high hydrophilicity [31]. Because the aqueous state has different effects on polymer properties [32], charged polymers exhibit much higher water adsorption and free water content than neutral and zwitterionic ones. The free water molecules around the polymer enhance protein resistance [33]. The denser the water layer, the higher the energy barrier to proteins [34]. Moreover, the various nanoarchitectures of different polymers act as a dual barrier against protein adsorption [35]. Nanostructured polymeric surfaces affect the orientation and order of protein adsorption [36].

The adsorption of biomolecules (e.g., proteins) affects the tribological properties of the implant [37]. Usually, the proteins adsorbed on the interfaces can reduce wear [38-40]. Sliding also promotes protein adsorption [41]. There are several types of proteins in vivo that affect the tribological properties in different ways [42, 43]. A pin-on-disk test showed that albumin yielded a lower friction coefficient than globulin. However, both exhibited a higher friction coefficient and wear rate than bovine synovial fluid [44]. Furthermore, kinematic conditions substantially affect the function of proteins. Under pure rolling conditions, albumin plays a critical role, whereas globulin is more important under high-speed slideroll conditions [45]. Other biomolecules within the synovial fluid also significantly affect the tribological properties. Polysaccharides are proposed to lower the friction coefficient [46]. As the main component of body fluids, hyaluronic acid (HA) influences the tribological properties and can prevent protein adsorption, especially inflammatory proteins [47, 48] The synergistic effect makes the friction regulation mechanism very complex. The polymer-modified surface further aggravates the complexity. For example, the interaction between protein and the polymer surface is affected by the behavior of the protein, which affects the lubrication synergy [49]. Different biomolecules affect each other because of their varying interactions with polymer molecules. The synergistic effects (e.g., adsorption, adsorption interaction, and regulation of surface characteristics after adsorption) of these constituents in synovial fluid make the tribological properties of polymermodified surfaces very complex. For water-based superlubricity systems, biomolecules induce a change from ultralow to high friction $[50,51]$. Thus, the synergistic regulation mechanism of biomolecules that determines the tribological performance of polymer-modified surfaces needs to be identified. 
In this study, we investigated the superlubricity properties of poly(vinylphosphonic acid) (PVPA)modified Ti6Al4V affected by different biomolecules. The real-time adsorption of different proteins on the PVPA coating was first evaluated. The mechanism of proteins that regulates the tribological properties of PVPA interfaces was proposed, considering the adsorption specificity of PVPA coatings. Then, the action of adsorption and further friction of polysaccharides were analyzed. Finally, the interactions between different biomolecules were explored. The synthetic effect of proteins and polysaccharides on the tribological properties of PVPA coatings was proposed. The proposed synthetic effect of biomolecule behavior on the tribological properties of PVPA coatings could serve as a guide for research on the superlubricity of hydrophilic polymers.

\section{Materials and methods}

\subsection{Materials}

PVPA (97\%) and phosphate-buffered saline (PBS, $\mathrm{pH}=7.2$ ) were provided by Sigma Aldrich (St. Louis, MO, USA). Albumin, globulin, and aggrecan (AGC), and HA were purchased from J\&K Chemicals (Beijing, China). Ti6Al4V (100 mm $\times 100 \mathrm{~mm}$ ) foils with a thickness of $1 \mathrm{~mm}$ were supplied by Goodfellow, Inc. (Cambridge, Uk). The titanium substrates were used in the modification, which was polished to smooth surfaces $(R a \approx 2 \mathrm{~nm}$ ). PTFE balls $(D \approx 6 \mathrm{~mm}$ ) with a roughness of approximately $280 \mathrm{~nm}$, were purchased from Taobao, Inc. All reagents mentioned above were used as received.

\subsection{Quartz crystal microbalance to explore the adsorption of biomolecules in real-time}

A quartz crystal microbalance (QCM)-D instrument (Q-sense E4 system, Biolin Scientific, Sweden) was used to measure the adsorption properties of the biomolecules. The changes in both resonance frequency $(\Delta f)$ and dissipation $(\Delta D)$ were simultaneously measured to calculate the adsorption of biomolecules on $\mathrm{TiO}_{2}$-coated quartz crystal sensors with and without PVPA coating. A phosphoric acid buffer was used as the base liquid, and the baseline frequency signal was measured until it stabilized. The solution with biomolecules was injected into the sample cell at a flow rate of $0.1 \mathrm{~mL} / \mathrm{min}$ at $37^{\circ} \mathrm{C}$. When the adsorbed film was rigid with low viscoelasticity, the Sauerbrey equation [52] was used to convert the frequency changes into adsorbed mass changes $(\Delta m)$ according to the following expression:

$$
\Delta m=-C \cdot \Delta f / n
$$

where $C$ is a constant of $17.7 \mathrm{ng} \cdot \mathrm{Hz}^{-1} \cdot \mathrm{cm}^{-2}$, which is related to the properties of the quartz crystal, and $n$ is the overtone of the oscillations.

\subsection{Universal microtribometer for the evaluation of tribological properties}

A universal microtribometer (UMT-3, BRUKER) was used to test the tribological behaviors of proteins and polysaccharides affecting the PVPA coating and bare Ti6Al4V. Another tribo-pair, the PTFE ball, was used to rub against Ti6Al4V. A reciprocating movement mode was chosen. The temperature during the sliding experiment was controlled at $37{ }^{\circ} \mathrm{C}$ to reflect the human body temperature. The initial contact pressure was approximately $25 \mathrm{MPa}$, whereas the average sliding speed was $12 \mathrm{~mm} / \mathrm{s}$.

\subsection{Microscopic observation of surface morphology after sliding}

The surface morphologies of wear scars and wear scratches after sliding were tested using a stereomicroscope (BX53M, OLYMPUS, Japan). Before the test, the tribo-pairs were washed with PBS. The test areas were amplified by at least 100 -fold to display the morphology. All the tests were repeated at least three times for each group.

\section{Results and discussion}

\subsection{Tribological properties of PVPA-modified Ti6A14V regulated by proteins based on interaction behaviors}

The friction experiments were performed in reciprocating mode with an initial load of $2.5 \mathrm{~N}$ at $37{ }^{\circ} \mathrm{C}$. Bare/PVPA-modified Ti6Al4V and PTFE balls were sampled as tribo-pairs. The reciprocating frequency was $2 \mathrm{~Hz}$, which corresponds to a sliding speed of 
$12 \mathrm{~mm} / \mathrm{s}$. PBS was used as the basic solution. Different protein solutions were prepared according to their content in synovial fluid. The experimental results of the PVPA-modified surfaces are shown in Fig. 1. Albumin and globulin were found to increase the friction coefficient of PVPA-modified Ti6Al4V. The proteins disturbed the superlubricity (friction coefficient $<0.01$ ) of the PVPA-PBS system. The influence of albumin was much more distinct than that of globulin. Unlike other surface topography after sliding, the protein solutions did not cause serious wear on the two tribo-pairs, but the friction coefficient was enhanced, as shown in Figs. 1(b) and 1(c). Thus, the larger friction coefficient was not caused by severe wear, and protein characteristics affect the friction coefficient.

The effect of proteins on the friction interfaces was determined by the surface properties of the tribo-pairs. The sliding experiment on bare Ti6Al4V,
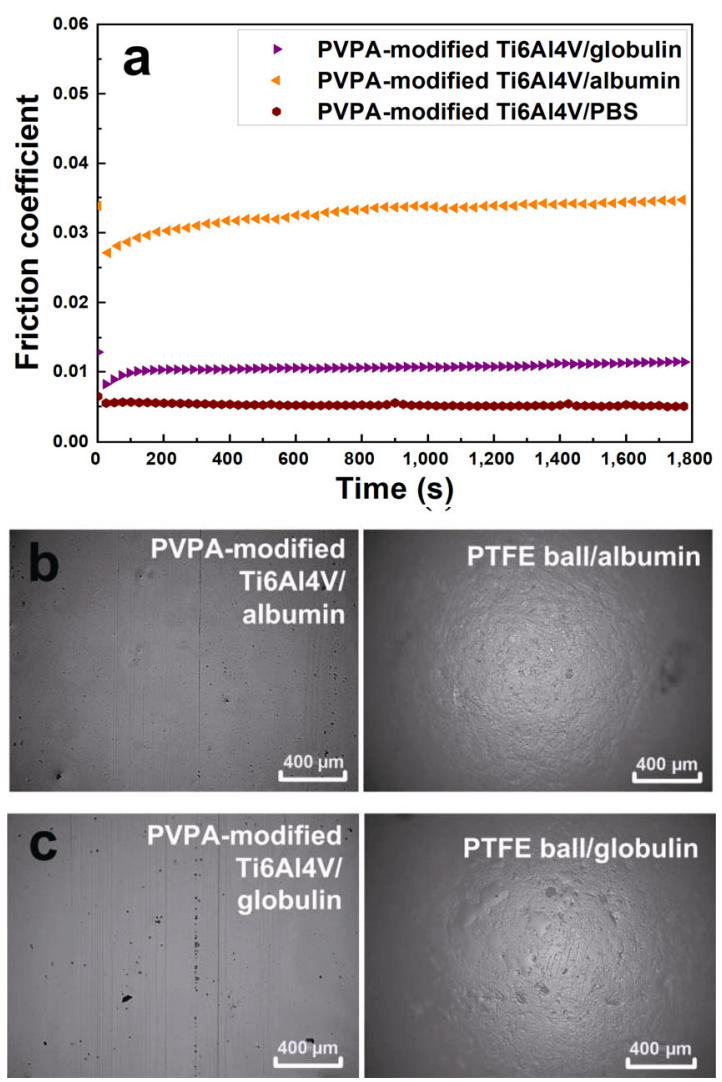

Fig. 1 Tribological properties of PVPA-modified Ti6Al4V with different protein solutions as a lubricant: (a) friction coefficient versus time and wear morphologies of two tribo-pairs lubricated by (b) albumin solution and (c) globulin solution. which has weak hydrophilicity, shows that the effects of proteins differed from those on PVPA-modified Ti6Al4V, as shown in Fig. 2. The friction coefficient of the albumin and globulin solutions showed a decreasing trend over time. No significant difference was observed between the two types of proteins. Thus, the different interactions between proteins and PVPA molecules increased the variations in friction, as shown in Fig. 1.

For the PVPA-modified Ti6Al4V, the PVPA coating itself can directly prevent asperity contact, and the influence of proteins on wear is negligible. However, the friction coefficient is determined by the surface hydration properties, especially for water-based superlubricity. Different proteins significantly affect the friction coefficient of PVPA-modified Ti6Al4V compared with bare Ti6Al4V. Because the polymer molecules of the modified surfaces can further regulate the behavior of proteins, various interactions can occur between proteins and PVPA molecules. Therefore, the protein adsorption behavior of the PVPA coating was further explored by QCM.

The protein solutions that acted as lubricants in the friction experiments were analyzed. Figure 3 shows the real-time adsorption process of albumin and globulin. When the PVPA-modified surfaces were immersed in protein solutions, the frequency decreased because of protein adsorption, as shown in Fig. 3(a1). Then, the PBS solution was added to rinse weakly adsorbed proteins from the surfaces. The frequency increased because of the few remaining stable proteins adsorbed on the surface, as shown in Fig. 3(a2). The frequency difference between

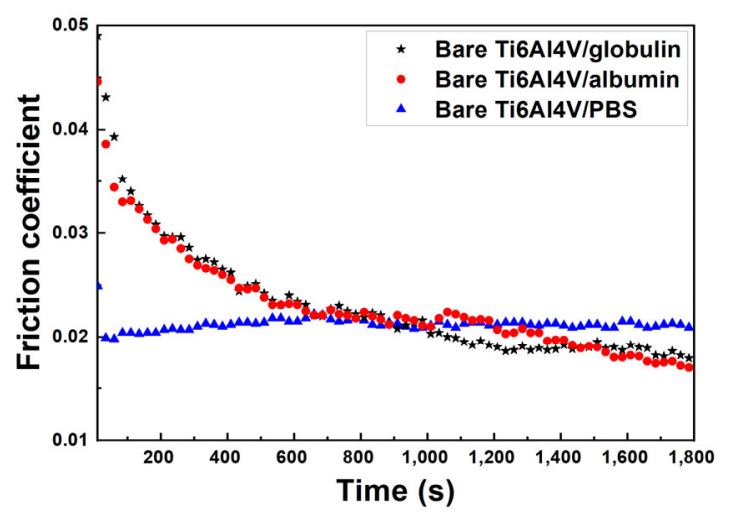

Fig. 2 Friction coefficient of bare Ti6Al4V with different protein solutions as a lubricant. 
adsorption and desorption was proportional to the stable adsorption mass. The calculation was performed using Eq. (2).

$$
m=-C \Delta f / n
$$

In Eq. (2), $C$ is a constant that depends on the intrinsic properties of quartz [53]. The value of $C$ is $17.7 \mathrm{ng} \cdot \mathrm{Hz}^{-1} \cdot \mathrm{cm}^{-2}$, and $n(1,3,5$, etc. $)$ is the overtone number. In this study, the third overtone $(n=3)$ was selected for the computational analysis [54]. Figure 3(a1) shows that more albumin was adsorbed on the PVPA surface compared with globulin. A high desorption ratio was observed in the globulin adsorption process, as shown in Fig. 3(a2). The stable adsorption capacity of globulin, which was obtained by subtracting the desorption mass from the initial adsorption mass, is small. According to the change in dissipation $(\Delta D)$, the dissipation of
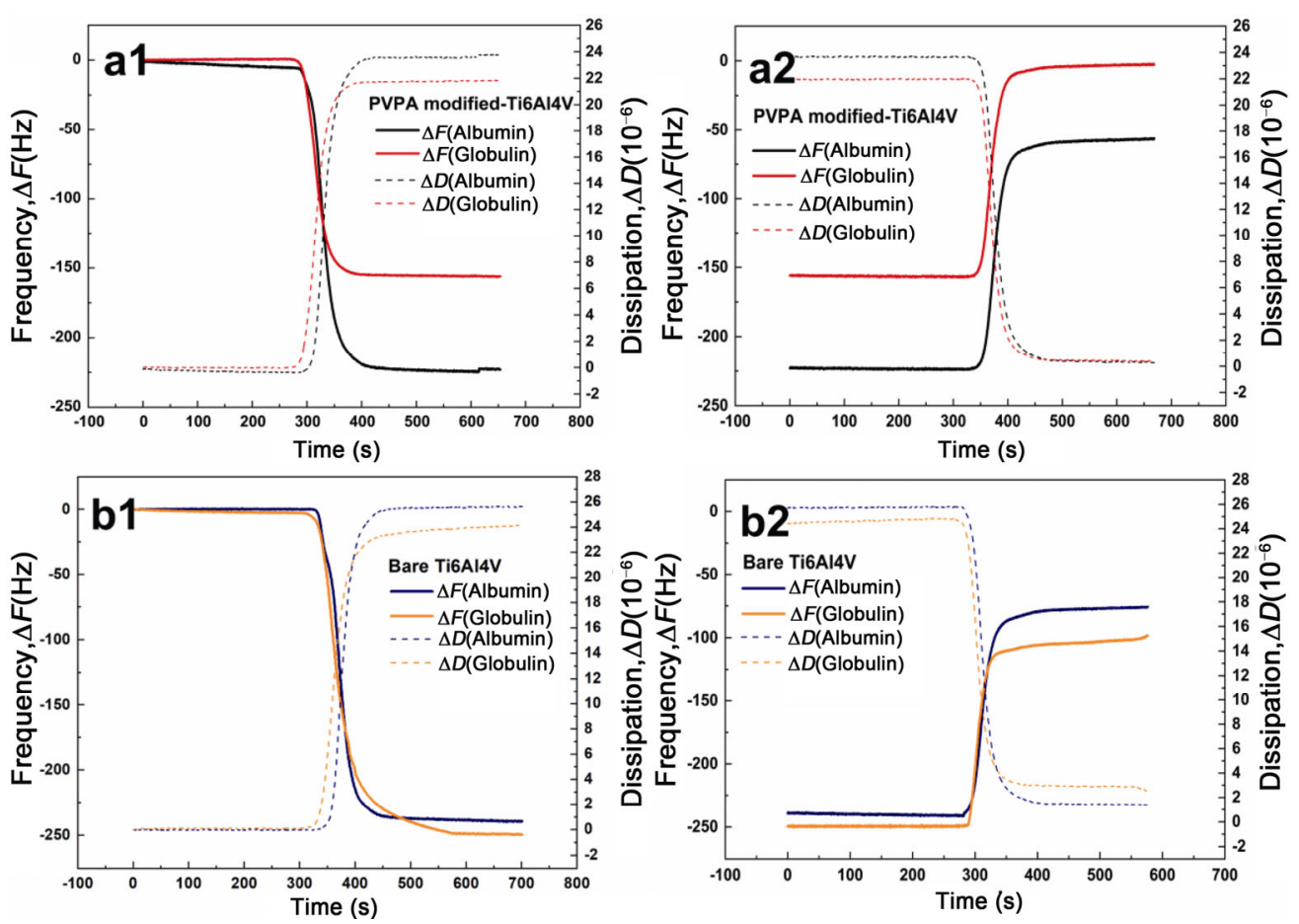

Fig. 3 Adsorption behaviors of proteins measured by QCM. (a) PVPA-modified surfaces: (a1) the adsorption process, (a2) the desorption process; (b) bare Ti6A14V surfaces: (b1) the adsorption process, (b2) the desorption process.

Table 1 Protein adsorption and desorption results.

\begin{tabular}{|c|c|c|c|c|c|c|}
\hline \multirow{2}{*}{ Protein } & \multicolumn{2}{|c|}{ Adsorption } & \multicolumn{2}{|c|}{ Desorption } & \multicolumn{2}{|c|}{ Net adsorption } \\
\hline & Mass $\left(\mathrm{ng} / \mathrm{cm}^{2}\right)$ & Thickness (nm) & Mass $\left(\mathrm{ng} / \mathrm{cm}^{2}\right)$ & Thickness (nm) & Mass $\left(\mathrm{ng} / \mathrm{cm}^{2}\right)$ & Thickness (nm) \\
\hline Albumin (PVPA) & 1315.70 & 13.1570 & 982.35 & 9.8235 & 333.35 & 3.3335 \\
\hline Globulin (PVPA) & 920.40 & 9.2040 & 905.65 & 9.0565 & 14.75 & 0.1475 \\
\hline Albumin (bare) & 1413.05 & 14.1305 & 964.65 & 9.6465 & 448.40 & 4.4840 \\
\hline Globulin (bare) & 1472.05 & 14.7205 & 908.60 & 9.0860 & 563.45 & 5.6345 \\
\hline
\end{tabular}

albumin and globulin is small enough $(\Delta D<\Delta f)$. Both the albumin and globulin adsorbed on the PVPA coating surface formed a rigid membrane, which is lightweight and thin, and was evenly distributed over the active area of the crystal membrane. The film thickness was obtained based on Eq. (3). The relative calculation results are shown in Table 1.

$$
h=-C(\Delta f / n) / 100
$$

For globulin, a very thin film of approximately $0.15 \mathrm{~nm}$ was formed on the PVPA-modified surfaces because of the unstable adsorption. Comparison with the results for bare Ti6Al4V in Fig. 3(b) and Table 1 show that the weak interaction between globulin and PVPA molecules is negligible. Therefore, the impact of the hydrogen properties of PVPA molecules is small. Figures 3(a1) and 3(a2) show that the adsorbed globulin molecules easily escaped 
from the surface. Most of the globulin molecules were removed when the PVPA coating was rinsed with PBS, which indicates the weak interaction between globulin and PVPA molecules. In other words, the dynamic motion of globulin on the PVPA coating surface is more violent compared with that of albumin. Tribological sliding behavior was not conducive to the formation of a globulin film. The coefficient of friction of the PVPA-modified surface in globulin solution increased slightly, as shown in Fig. 1. However, a more stable albumin film formed on the PVPA-modified surface, with a thickness of approximately $3.3 \mathrm{~nm}$, which is 22 times that of globulin. The large stable albumin adsorption considerably affects the surface characteristics of the PVPA coating. The superlubricity was observed to have disappeared. Compared with the results of bare $\mathrm{Ti} 6 \mathrm{Al} 14 \mathrm{~V}$, whose interaction with protein is stronger, as shown in Fig. 3(b) and Table 1, the adsorbed albumin almost entirely covered the PVPA surface. A strong interaction was observed between the albumin and PVPA molecules, which has a large influence on friction properties.

In conclusion, protein can affect the tribological properties of PVPA-modified systems. Their adsorption disturbs the hydration characteristics of PVPA molecules. Therefore, the stronger the interaction between albumin molecules and PVPA molecules is, the larger the friction coefficient is. When the protein interacts with PVPA-modified systems, the superlubricity disappears. The dynamic behavior of proteins on the PVPA-modified surfaces is more evident than that on bare Ti6Al4V because of the polymer molecules, which is beneficial for the requirement of a low friction coefficient.

\subsection{Tribological properties of PVPA-modified} Ti6Al4V regulated by polysaccharides based on the interaction behaviors

Apart from proteins, another important constituent in synovial fluid is polysaccharides. These have different effects on friction compared with proteins. Polysaccharide solutions were prepared as lubricants by adding the polysaccharides AGC and HA to PBS according to their content in synovial fluid. The resulting friction coefficients are shown in Fig. 4(a). As polysaccharides were added, the friction coefficient increased compared with that of the PVPA-PBS system, indicating that the surface characteristics of the PVPA coating were also affected. The superlubricity of the PVPA-PBS system disappeared when AGC was added, while HA did not eliminate the superlubricity. This phenomenon supports the influence of proteins mentioned above. The protein-related structures in AGC is the reason why it has a greater effect on the superlubricity of the PVPA coating compared with HA. However, even for the AGC solution, the friction coefficient was only slightly elevated. Compared with the friction coefficients in the case of proteins (Fig. 1(a)), the influence of polysaccharides on the friction coefficient was smaller. The wear on the two tribo-pairs was also negligible, as shown in Figs. 4(b) and 4(c).

The high hydration characteristics and similar
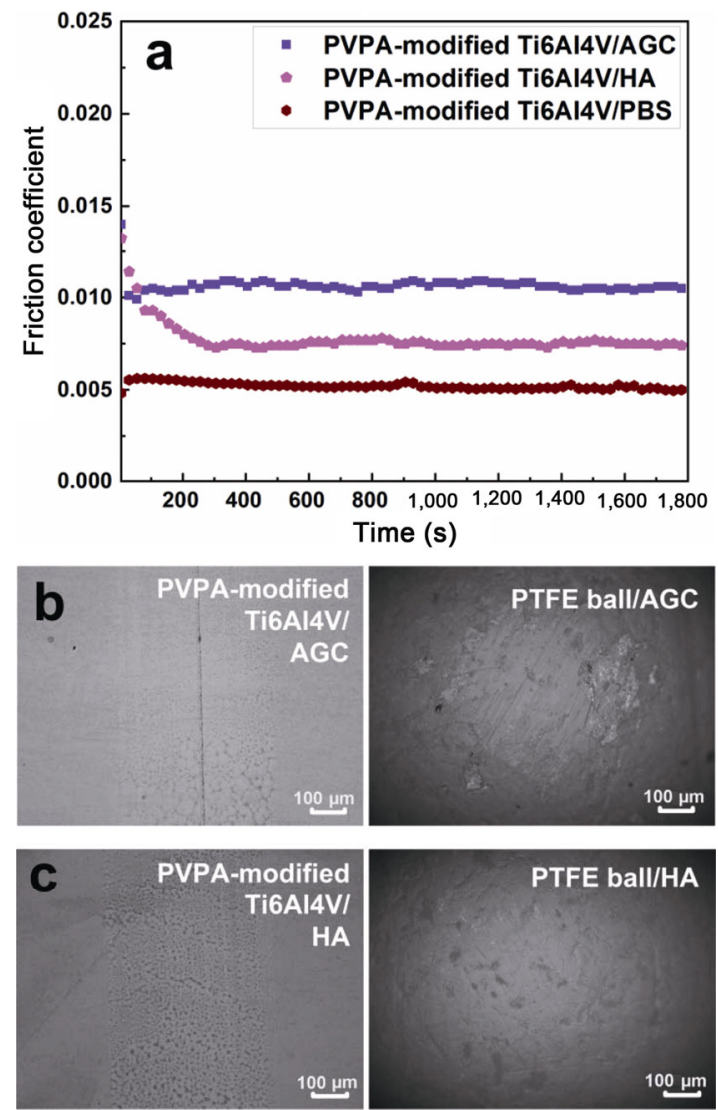

Fig. 4 Tribological properties of PVPA-modified Ti6A14V with different polysaccharide solutions as the lubricant: (a) friction coefficient versus time and wear morphologies of the two tribo-pairs lubricated by (b) AGC solution and (c) HA solution. 
hydrophilic polymer structures of polysaccharides are the main reasons for their negligible effect on the hydrophilic polymer coating. The lubrication action of polysaccharides was proved by the behavior observed in bare Ti6Al4V, as shown in Fig. 5. The friction coefficient values with HA and AGC as lubricants were lower than those with PBS as a lubricant. This is because the water film that formed on the interfaces in water-based lubrication is important for friction. During the sliding experiment, the brush-like adsorption film formed by the AGC and HA molecules on bare Ti6Al4V surfaces improved the direct asperity contact and hydration properties. The higher the hydration characteristics and HA adsorption, the lower the friction coefficient compared with that of the AGC.

However, why do the strong hydrophilic polymers of polysaccharides not promote the decrease in the friction coefficient of PVPA-modified surfaces? If there is a complete lubricating film, the viscous resistance regulated by the viscosity of lubricants is critical when the friction coefficient is very low; for example, within the range of superlubricity. The viscosity of different polysaccharide lubricants was tested, and the results are shown in Fig. 6. The viscosity of the HA solution was approximately $7.317 \times 10^{-3} \mathrm{~Pa} \cdot \mathrm{s}$, which is higher than that of AGC (approximately $0.9134 \times 10^{-3} \mathrm{~Pa} \cdot \mathrm{s}$ ). The viscosity of the PBS solution was approximately $0.695 \times 10^{-3}$ $\mathrm{Pa} \cdot \mathrm{s}$, which is the lowest. Then, the theoretical hydrodynamic film thickness was calculated (Fig. S1(Electronic Supplementary Material, ESM)). For the PVPA-modified Ti6Al4V, the thickness of the

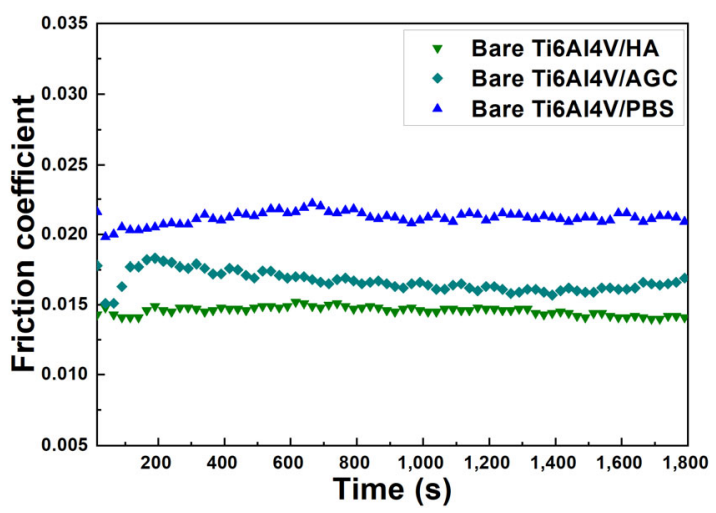

Fig. 5 Friction coefficient of bare Ti6Al4V with different polysaccharide solutions as a lubricant. lubricating film after polysaccharide adsorption was less than $6 \mathrm{~nm}$. Irrespective of whether the lubrication film thickness increased or decreased, the film thickness ratio was less than 0.03 (Fig. S1(ESM))). This indicates that regardless of the difference in viscosity, the lubrication condition is always in the boundary lubrication state. Therefore, the different interactions between polysaccharides and PVPA molecules were explored to identify the factors influencing the friction performance.

The interfacial behaviors of the polysaccharides were explored by QCM. The polysaccharide solutions that acted as lubricants in the friction experiments were used. Figure 7 shows the adsorption process of solutions with AGC and HA. The values of the adsorption mass and film thickness were calculated using Eqs. (2) and (3), respectively; the results are listed in Table 2. No AGC adsorption or desorption behaviors were observed on the PVPA-modified surfaces, as shown in Fig. 7(a), which confirmed the absence of interactions between the AGC and PVPA molecules. The tube brush-like structure of AGC molecules promoted the formation of a strongly hydrated sheath $[55,56]$. Because of the lower content of AGC molecules in the solution, an AGC hydration microgel was speculated to have formed and distributed within the solution. The water around the microgel showed adsorption inertia, even for bare Ti6Al4V, as shown in Fig. 7(b). However, the existence of the AGC hydration microgel increased the viscosity of the lubricant. The slightly increased friction coefficient is related to the viscous resistance of the lubricant because

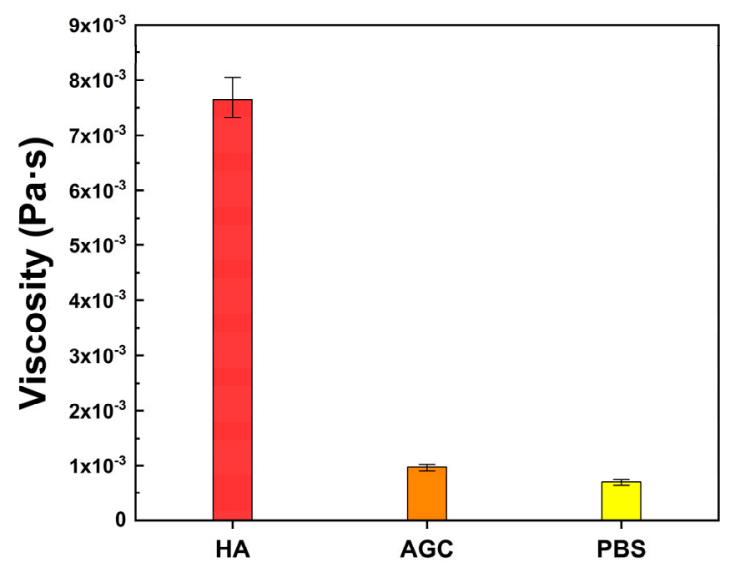

Fig. 6 Viscosity of different polysaccharide solutions. 
there was no interaction between the AGC and PVPA molecules. A completely different phenomenon was observed in the case of HA. The interaction between HA and PVPA molecules was very active. HA molecules showed a high dynamic movement. Figure 7(a1) shows the adsorption capacities when the PVPA-modified surfaces were immersed in the HA solution. Almost all the adsorbed HA molecules were desorbed from the PVPA molecules after PBS was added to the adsorbed surfaces, as shown in Fig. 7(a2). Stably adsorbed HA molecules were not observed on the modified surfaces. Owing to the high hydration properties of HA, a hydration lubrication layer formed on the PVPA interfaces even when only a small amount of HA was absorbed.
Correspondingly, since the hydration lubrication layer was formed on the PVPA interfaces, superlubricity can be obtained similar to that lubricated by pure PBS. However, the viscosity of the HA solution was higher than that of the pure PBS solution. The synergistic effect of the two factors leads to a slightly larger final friction coefficient.

Polysaccharides tend to be adsorbed at low levels on PVPA-modified surfaces because of their hydrophilic polymer characteristics. The calculated film thickness confirmed that they were in the boundary lubrication range. Therefore, because of their similar hydrophilic potential, an effective hydration layer was formed on the friction interfaces, regardless of whether the polysaccharide molecules were adsorbed
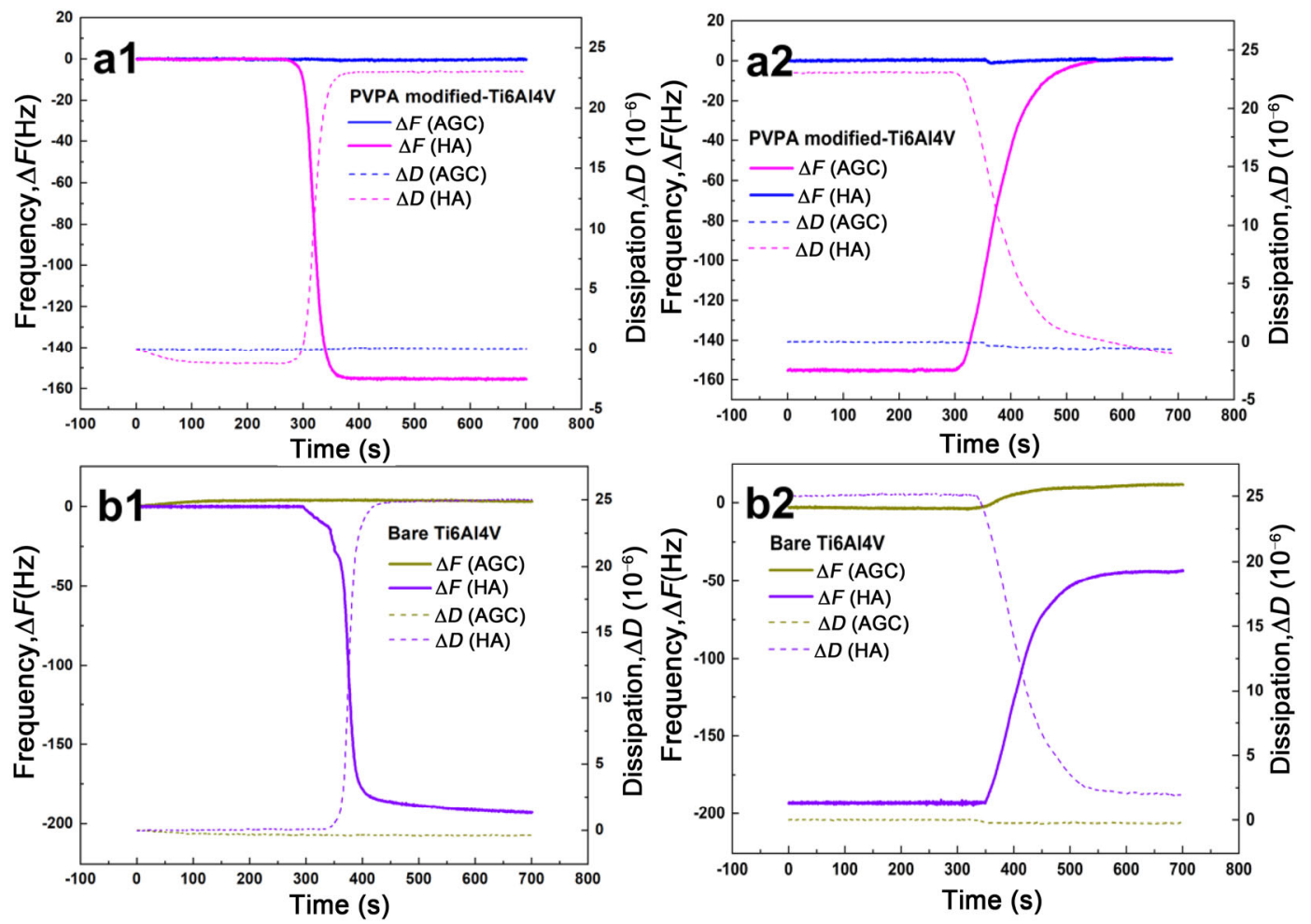

Fig. 7 Polysaccharide adsorption is measured by QCM. (a) PVPA-modified Ti6Al4V: (a1) the adsorption process, (a2) the desorption process; (b) bare Ti6Al4V: (b1) the adsorption process, (b2) the desorption process.

Table 2 Polysaccharide adsorption and desorption results.

\begin{tabular}{|c|c|c|c|c|c|c|}
\hline \multirow{2}{*}{ Polysaccharide } & \multicolumn{2}{|c|}{ Adsorption } & \multicolumn{2}{|c|}{ Desorption } & \multicolumn{2}{|c|}{ Net adsorption } \\
\hline & $\operatorname{Mass}\left(\mathrm{ng} / \mathrm{cm}^{2}\right)$ & Thickness (nm) & $\operatorname{Mass}\left(\mathrm{ng} / \mathrm{cm}^{2}\right)$ & Thickness (nm) & Mass $\left(\mathrm{ng} / \mathrm{cm}^{2}\right)$ & Thickness (nm) \\
\hline AGC (PVPA) & 0 & 0 & 0 & 0 & 0 & 0 \\
\hline HA (PVPA) & 917.45 & 9.1745 & 914.50 & 9.1450 & 2.95 & 0.0295 \\
\hline AGC (bare) & 17.70 & 0.1770 & 11.80 & 0.1180 & 5.90 & 0.0590 \\
\hline HA (bare) & 1138.70 & 11.3870 & 882.05 & 8.8205 & 256.65 & 2.5665 \\
\hline
\end{tabular}


or not, and the superlubricity did not disappear. Furthermore, the lower the viscosity is, the smaller the friction coefficient is. The excellent resistance of polysaccharide biomolecules to PVPA coatings is beneficial for the application of PVPA in vivo.

\subsection{Synergistic regulation mechanism of biomo- lecule adsorption-friction properties}

The tribological properties of implants in vivo are caused by the combination of all types of biomolecules. To determine the synergy, albumin, globulin, AGC, and HA were combined in PBS according to their relative content in synovial fluid. The friction experiments were performed using the same parameters as above. The friction coefficients are shown in Fig. 8. For bare Ti6Al4V, the friction coefficient in the mixed solution was approximately 0.030, which is closer to that of the protein solution as a lubricant than that of the polysaccharide as a lubricant. In other words, the interface properties are mainly controlled by proteins rather than the synergistic action of different biomolecules. This is because protein adsorption is prioritized over polysaccharide adsorption when bare Ti6Al4V is immersed in the mixed solution. This is evident in the comparison of the dynamic adsorption behavior of different biomolecules in Figs. 3(b) and 7(b). However, the tribological properties of PVPA surfaces become more complex because of the interaction with different polymer molecules. The friction coefficient of the PVPA-modified Ti6Al4V in the mixed solution was approximately 0.017, which is between those of albumin, globulin, AGC, and HA. For the PVPA molecules, a preferential interaction occurred between proteins and PVPA molecules, as shown by the QCM measurement above (Figs. 3(a) and 7(a)). Notably, because the protein molecules displayed high dynamic movement owing to the higher protein resistance of the PVPA coating, the adsorption film was unstable. HA molecules were also found to be adsorbed on the PVPA coatings. The properties of the interfaces were regulated by both proteins and polysaccharides. Therefore, the friction coefficient of the surface lubricated by the mixed solution was lower than that of the surface lubricated by the protein solution and higher than that of the surface lubricated by polysaccharide solution.

Additional comparison experiments were performed to identify the mechanism of synergy. The competing interactions between biomolecules and PVPA molecules and the mutual responses of different biomolecules were explored by solution mixing during sliding. During the sliding experiment, when the protein solution (consisting of albumin and globulin) was added to the polysaccharide lubricant (consisting of AGC and HA), the friction coefficient suddenly increased from 0.014 to 0.023 , as shown by the magenta line in Fig. 9(a). This indicates that proteins selectively interacted with PVPA molecules, and the adsorption of polysaccharide molecules was negligible and unstable. When protein molecules were added to the lubricant, the interaction between the polysaccharide and PVPA molecules could be easily replaced by the interaction between the protein and PVPA molecules, thus covering
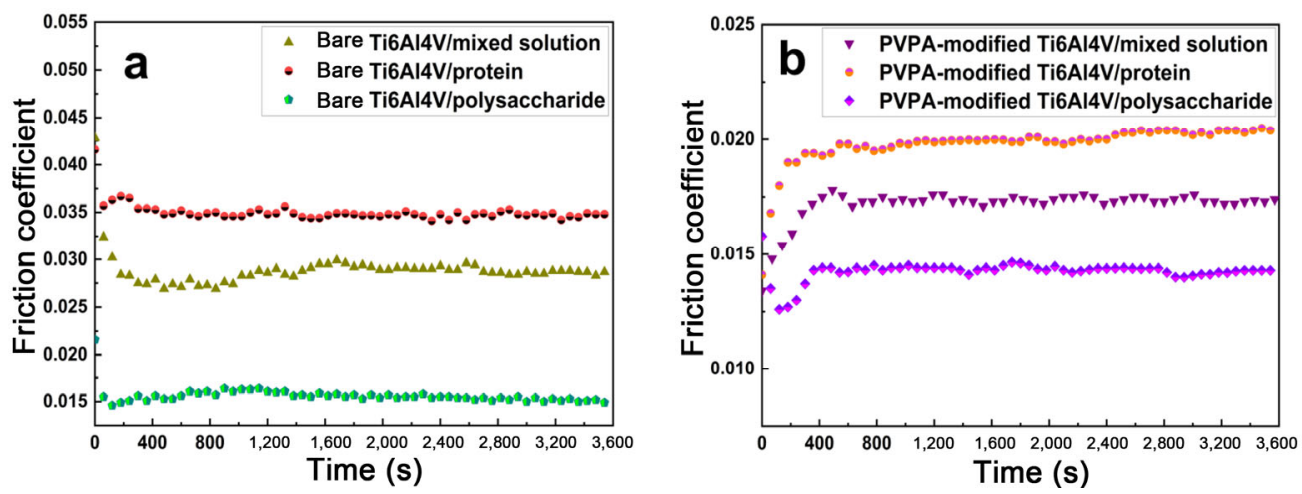

Fig. 8 Friction coefficient versus time for (a) bare Ti6Al4V and (b) PVPA-modified Ti6Al4V in mixed solution, proteins, and polysaccharides. 
the PVPA coating interface with the protein. The protein adsorption film, which causes more serious damage to the hydration characteristics of interfaces, determines the tribological properties. Therefore, the friction coefficient increased. However, when the experimental process was reversed and the polysaccharide solution was added to the protein solution during sliding, the friction coefficient slightly decreased, as shown by the cyan line in Fig. 9(a). This phenomenon not only proves the critical role of protein, but also emphasizes the dynamic behavior of protein molecules on the PVPA coating interfaces; that is, the PVPA coating is more protein-resistant (especially for globulin) than bare Ti6Al4V. Some HA molecules can partially replace the adsorbed protein molecules and reduce protein adsorption. The friction coefficient was also slightly reduced.

For different protein molecules, the interaction given the highest priority with PVPA molecules is that with albumin, as shown in Fig. 9(b). The friction state under globulin lubrication is easily destroyed by albumin, as indicated by the red line in Fig. 9(b). The friction coefficient increased when the albumin solution was added to the globulin solution during sliding. Meanwhile, the interaction between albumin and PVPA molecules is strong. The friction coefficient was not altered by globulin, as shown by the gray line in Fig. 9(b). However, no complete adsorption was observed between the polysaccharides, as shown in Fig. 9(c). The friction states were not affected by the mixed solution of HA and AGC. The inherent hydration and polymer structure of polysaccharide biomacromolecules are advantageous because of the stronger resistance of PVPA molecules to the polysaccharides, such as AGC; it is beneficial for the formation of the hydration layer. The adsorption capacity of polysaccharides is weak, and their influence on the tribological properties is attributed to hydration and viscosity. The ultralow friction was maintained.

The competitive adsorption of different biomolecules was verified by QCM experiments, as shown in Fig. 10 . Following the stable adsorption of polysaccharide
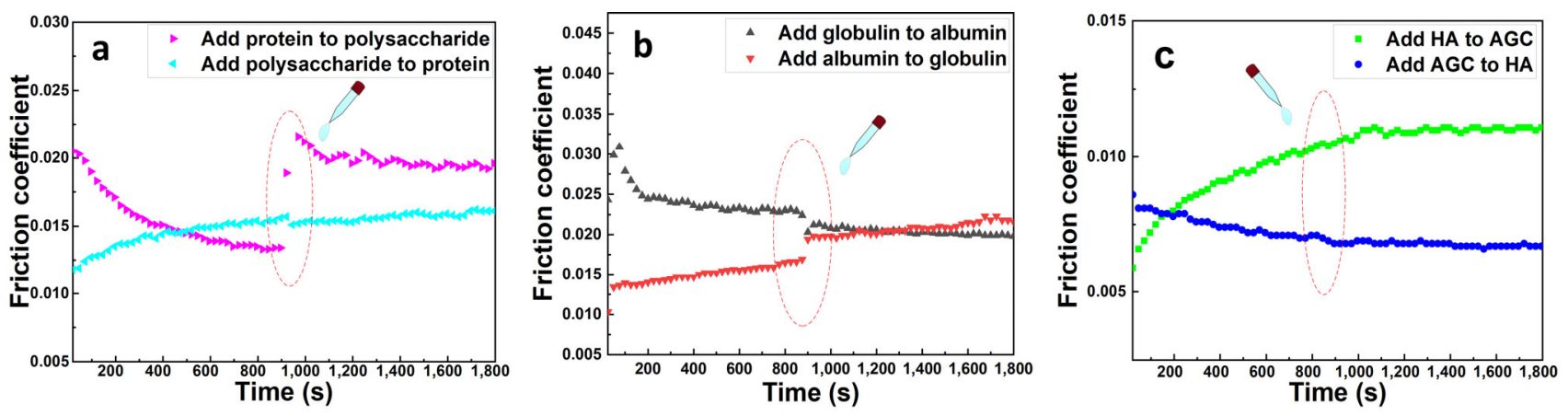

Fig. 9 Comparison of interaction behaviors of different biomolecules: (a) protein and polysaccharide, (b) albumin and globulin, and (c) AGC and HA.
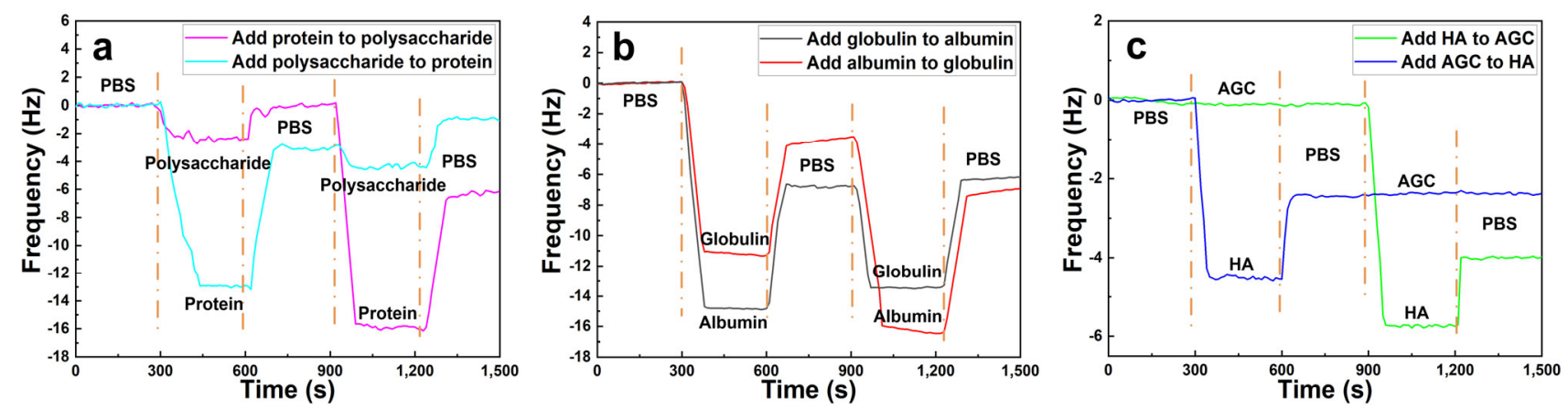

Fig. 10 Competitive adsorption measured by QCM: (a) protein and polysaccharide, (b) albumin and globulin, and (c) AGC and HA. 
molecules, the introduction of protein molecules further reduced the frequency, as shown by the magenta line in Fig. 10(a); this means that adsorption of protein molecules can continue on the PVPA coating. Therefore, the friction coefficient was significantly increased when the protein solution was added to the polysaccharide solution. In other words, proteins display stronger and more competitive adsorption behavior compared with polysaccharides. The vibration frequency of QCM increased after the introduction of polysaccharides, as shown by the cyan line in Fig. 10(a). This means that adsorption of polysaccharide molecules not only continued, but also reduced the adsorption of protein molecules. The slight change in adsorption had little effect on the friction coefficient.

Similar QCM comparison experiments on globulin/ albumin mixing were performed, as shown in Fig. 10(b). It was found that the adsorption of albumin molecules on the PVPA coating continued after stable globulin adsorption, as shown by the red line in Fig. 10(b). That is, the adsorption of albumin is more competitive than that of globulin. This is the reason for the increase in friction coefficient when albumin was added to the globulin solution, as shown by the red line in Fig. 9(b). In contrast, the adsorption of globulin molecules ceased after the stable adsorption of albumin molecules, and caused partial desorption of the adsorbed albumin molecules, as shown by the gray line in Fig. 10(b). When the globulin was added to the albumin solution, the friction coefficient decreased slightly, as shown by the gray line in Fig. 9(b).

For the QCM comparison experiment on AGC/ HA mixing, it was found that AGC molecules could not be adsorbed on the PVPA surface, while HA molecules were steadily adsorbed. When AGC was added to the HA solution, as shown by the blue line in Fig. 10(c), the stable adsorption state of HA remained unchanged. The friction coefficient also did not change. However, when HA was added to AGC, a large number of HA molecules are continuously adsorbed, as shown by the green line in Fig. 10(c). Despite competitive adsorption, a sudden change in the friction coefficient did not occur owing to the similar strong hydrophilic property of polysaccharide molecules.
These results show that both proteins and polysaccharides affect friction, but through different mechanisms. Proteins are more likely to adsorb on the PVPA coatings, especially albumin, compared with polysaccharides. Then, the protein film that formed determined the friction results. For polysaccharides, the high hydrophilicity and unstable dynamic adsorption had a small impact on the adsorption film. Both hydration and viscosity affected the friction coefficient. Polysaccharide molecules reduced the adsorption between proteins and PVPA molecules, and friction was affected by their synergistic action. Therefore, when the mixed solution was used as a lubricant, despite the preferential adsorption of proteins on the PVPA coating, some polysaccharide molecules can replace the adsorbed protein molecules, thus reducing the amount of protein adsorption, as shown in Fig. 11. With the comprehensive action of biomolecules, the friction coefficient under the lubrication of the mixed solution is 0.017 .

\section{Conclusions}

The superlubricity properties of PVPA-modified Ti6Al4V were influenced by biomolecules in different ways. Compared with polysaccharides, proteins displayed stronger interactions with PVPA molecules, which promoted protein film formation. The stronger interaction between albumin and PVPA led to a larger friction coefficient compared with that of globulin. Because of their hydrophilic properties and polymer structure, polysaccharides display high adsorption dynamics, leading to unstable interactions with PVPA molecules. In particular, no AGC molecule adsorption occurred on the PVPAmodified surfaces. Because the interaction between polysaccharides and PVPA is negligible, the tribological

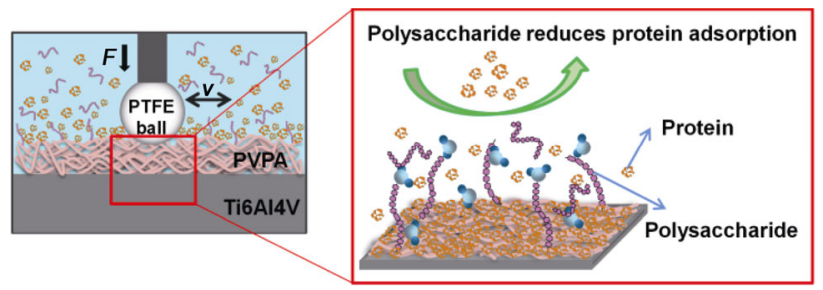

Fig. 11 Synergistic action of different biomolecules on friction. 
behavior is slightly affected by the polysaccharide adsorption film. The effective hydration lubrication layer still determines the superlubricity. The lower viscosity of the polysaccharide solution corresponds to a smaller friction coefficient. There is competition for interaction among different biomolecules. Polysaccharides have the potential to reduce protein adsorption. The proposed synthetic effect of biomolecule behaviors on the tribological properties of PVPA coatings can serve as a guide for research on the superlubricity of hydrophilic polymers.

\section{Acknowledgements}

This work was supported by the National Natural Science Foundation of China (51705010, 51875303), the Beijing Natural Science Foundation (3192003), the General Project of Science and Technology Plan from Beijing Educational Committee (KM201810005013), the Tribology Science Fund of State Key Laboratory of Tribology (STLEKF16A02, SKLTKF19B08), the training program of Rixin talent and outstanding talent from Beijing University of Technology.

Electronic Supplementary Material: Supplementary material is available in the online version of this article at https: //doi.org/10.1007/s40544-020-0441-9.

Open Access This article is licensed under a Creative Commons Attribution 4.0 International Li-cense, which permits use, sharing, adaptation, distribution and reproduction in any medium or for-mat, as long as you give appropriate credit to the original author(s) and the source, provide a link to the Creative Commons licence, and indicate if changes were made.

The images or other third party material in this article are included in the article's Creative Commons licence, unless indicated otherwise in a credit line to the material. If material is not in-cluded in the article's Creative Commons licence and your intended use is not permitted by statutory regulation or exceeds the permitted use, you will need to obtain permission directly from the copyright holder.

To view a copy of this licence, visit http://creativecommons.org/licenses/by/4.0/.

\section{References}

[1] Meng Y G, Xu J, Jin Z M, Braham P, Hu Y Z. A review of recent advances in tribology. Friction 8(2): 221-300 (2020)

[2] Philip J T, Mathew J, Kuriachen B. Tribology of $\mathrm{Ti}_{6} \mathrm{Al}_{4} \mathrm{~V}$ : A review. Friction 7(6): 497-536 (2019)

[3] Henriques B, Sampaio M, Buciumeanu M, Souza J C M, Gomes J R, Silva F, Carvalho O. Laser surface structuring of $\mathrm{Ti}_{6} \mathrm{Al}_{4} \mathrm{~V}$ substrates for adhesion enhancement in $\mathrm{Ti}_{6} \mathrm{Al}_{4} \mathrm{~V}-\mathrm{PEEK}$ joints. Mater Sci Eng: $C$ 79: 177-184 (2017)

[4] Luo Y, Chen W W, Tian M C, Teng S H. Thermal oxidation of $\mathrm{Ti}_{6} \mathrm{Al}_{4} \mathrm{~V}$ alloy and its biotribological properties under serum lubrication. Tribol Int 89: 67-71 (2015)

[5] Budinski K G. Tribological properties of titanium alloys. Wear 151(2): 203-217 (1991)

[6] Wang W, He Y Y, Zhao J, Mao J Y, Hu Y T, Luo J B. Optimization of groove texture profile to improve hydrodynamic lubrication performance: Theory and experiments. Friction 8(1): 83-94 (2020)

[7] Luo J B, Zhou X. Superlubricitive engineering-Future industry nearly getting rid of wear and frictional energy consumption. Friction 8(4): 643-665 (2020)

[8] Zhang C X, Liu Z F, Liu Y H, Cheng Q, Yang C B, Cai L $\mathrm{G}$. Investigation of the mechanisms for stable superlubricity of poly (vinylphosphonic acid) (PVPA) coatings affected by lubricant. Friction 4(4): 303-312 (2016)

[9] Wang W, Xie G X, Luo J B. Superlubricity of black phosphorus as lubricant additive. ACS Appl Mater Interfaces 10(49): 43203-43210 (2018)

[10] Nilsson D, Prakash B. Static/dynamic friction and wear of some selected polymeric materials for conformal tribo-pairs under boundary lubrication conditions. Friction 1(3): 232-241 (2013)

[11] Liao T T, Zhang T F, Li S S, Deng Q Y, Wu B J, Zhang Y Z, Zhou Y J, Guo Y B, Leng Y X, Huang N. Biological responses of Diamond-Like Carbon (DLC) films with different structures in biomedical application. Mater Sci Eng: C 69: 751-759 (2016)

[12] Luo Y, Yang T, Liu Q Z. Friction and wear of diamond-like carbon film deposited on CoCrMo alloy under different lubrication. Int $J$ Mater Res 107(7): 631-636 (2016)

[13] Ueda M, Silva M M, Otani C, Reuther H, Yatsuzuka M, Lepienski C M, Berni L A. Improvement of tribological properties of $\mathrm{Ti}_{6} \mathrm{Al}_{4} \mathrm{~V}$ by nitrogen plasma immersion ion implantation. Surf Coat Technol 169: 408-410 (2003)

[14] Zhang C X, Liu Y H, Wen S Z, Wang S. Poly (vinylphosphonic acid) (PVPA) on titanium alloy acting 
as effective cartilage-like Superlubricity coatings. $A C S$ Appl Mater Interfaces 6(20): 17571-17578 (2014)

[15] Durdu S, Usta M. The tribological properties of bioceramic coatings produced on $\mathrm{Ti}_{6} \mathrm{Al}_{4} \mathrm{~V}$ alloy by plasma electrolytic oxidation. Ceram Int 40(2): 36273635 (2014)

[16] Wang H, Lin C C, Zhang X R, Lin K L, Wang X D, Shen S G. Mussel-inspired Polydopamine coating: A general strategy to enhance Osteogenic differentiation and Osseointegration for diverse implants. ACS Appl Mater Interfaces 11(7): 7615-7625 (2019)

[17] Milner P E, Parkes M, Puetzer J L, Chapman R, Stevens M M, Cann P, Jeffers J R T. A low friction, biphasic and boundary lubricating hydrogel for cartilage replacement. Acta Biomater 65: 102-111 (2018)

[18] Han L B, Xiang L, Zhang J W, Chen J S, Liu J F, Yan B, Zeng $\mathrm{H}$ B. Biomimetic lubrication and surface interactions of dopamine-assisted Zwitterionic polyelectrolyte coatings. Langmuir 34(38): 1159311601 (2018)

[19] Ye T T, Deng Q Y, Ma D L, Li P C, Huang B, Leng Y X, Zhao Y C. Effect of grafted poly [2-methacryloyloxyethyl phosphorylcholine (MPC)] on tribological properties of ultra-high molecular weight polyethylene (UHMWPE). Int J Mod Phys B 33(1-3): 1940056 (2019)

[20] Ghosh S, Abanteriba S, Wong S, Houshyar S. Selective laser melted titanium alloys for hip implant applications: Surface modification with new method of polymer grafting. J Mech Behav Biomed Mater 87: 312-324 (2018)

[21] Iuster N, Tairy O, Driver M J, Armes S P, Klein J. Cross-linking highly lubricious Phosphocholinated polymer brushes: Effect on surface interactions and frictional behavior. Macromolecules 50(18): 7361-7371 (2017)

[22] Buxadera-Palomero J, Calvo C, Torrent-Camarero S, Gil F J, Mas-Moruno C, Canal C, Rodríguez D. Biofunctional polyethylene glycol coatings on titanium: An in vitro-based comparison of functionalization methods. Coll Surf B: Biointerf 152: 367-375 (2017)

[23] Gunkel G, Weinhart M, Becherer T, Haag R, Huck W T S. Effect of polymer brush architecture on Antibiofouling properties. Biomacromolecules 12(11): 4169-4172 (2011)

[24] Zeng G H, Ogaki R, Meyer R L. Non-proteinaceous bacterial adhesins challenge the antifouling properties of polymer brush coatings. Acta Biomater 24: 64-73 (2015)

[25] Harris L G, Tosatti S, Wieland M, Textor M, Richards R G. Staphylococcus aureus adhesion to titanium oxide surfaces coated with non-functionalized and peptidefunctionalized poly(L-lysine)-grafted-poly (ethylene glycol) copolymers. Biomaterials 25(18): 4135-4148 (2004)

[26] Wang K, Xiong D S. Construction of lubricant composite coating on $\mathrm{Ti}_{6} \mathrm{Al}_{4} \mathrm{~V}$ alloy using micro-arc oxidation and grafting hydrophilic polymer. Mater Sci Eng: C 90: 219-226 (2018)

[27] Zhang C X, Liu Z F, Liu Y H, Ren J, Cheng Q, Yang C B, Cai L G. Novel tribological stability of the superlubricity poly (vinylphosphonic acid) (PVPA) coatings on $\mathrm{Ti}_{6} \mathrm{Al}_{4} \mathrm{~V}$ : Velocity and load independence. Appl Surf Sci 392: 19-26 (2017)

[28] Zhang C X, Liu Y H, Liu Z F, Zhang H Y, Cheng Q, Yang C B. Regulation mechanism of salt ions for Superlubricity of hydrophilic polymer cross-linked networks on $\mathrm{Ti}_{6} \mathrm{Al}_{4} \mathrm{~V}$. Langmuir 33(9): 2133-2140 (2017)

[29] Murakami T, Yarimitsu S, Nakashima K, Sawae Y, Sakai $\mathrm{N}$. Influence of synovia constituents on tribological behaviors of articular cartilage. Friction 1(2): 150-162 (2013)

[30] Liu S Z, Zhang Q, Han Y, Sun Y L, Zhang Y F, Zhang H Y. Bioinspired surface functionalization of titanium alloy for enhanced lubrication and bacterial resistance. Langmuir 35(40): 13189-13195 (2019)

[31] Kyomoto M, Moro T, Saiga K, Hashimoto M, Ito H, Kawaguchi H, Takatori Y, Ishihara K. Biomimetic hydration lubrication with various polyelectrolyte layers on cross-linked polyethylene orthopedic bearing materials. Biomaterials 33(18): 4451-4459 (2012)

[32] Tran T, Lin C, Chaurasia S, Lin H Q. Elucidating the relationship between states of water and ion transport properties in hydrated polymers. J Memb Sci 574: 299-308 (2019)

[33] Wu J, Lin W F, Wang Z, Chen S F, Chang Y. Investigation of the hydration of Nonfouling material Poly(sulfobetaine methacrylate) by low-field nuclear magnetic resonance. Langmuir 28(19): 7436-7441 (2012)

[34] Xiao S W, Ren B P, Huang L, Shen M X, Zhang Y X, Zhong $M$ Q, Yang J T, Zheng J. Salt-responsive zwitterionic polymer brushes with anti-polyelectrolyte property. Curr Opin Chem Eng 19(4): 86-93 (2018)

[35] Endoh M K, Morimitsu Y, Salatto D, Huang Z X, Sen M N, Li W Y, Meng Y Z, Thanassi D G, Carrillo J M Y, Sumpter B G, et al. Protein resistance driven by polymer Nanoarchitecture. ACS Macro Lett 8(9): 1153-1159 (2019)

[36] Firkowska-Boden I, Zhang X Y, Jandt K D. Controlling protein adsorption through nanostructured polymeric surfaces. Adv Healthc Mater 7(1): 1700995 (2018)

[37] Wu B J, Deng Q Y, Leng Y X, Wang C M, Huang N. Characterization of adsorption and lubrication of synovial fluid proteins and HA on DLC joint bearings 
surface. Surf Coat Technol 320: 320-332 (2017)

[38] Hakala T J, Laaksonen P, Saikko V, Ahlroos T, Helle A, Mahlberg R, Hähl H, Jacobs K, Kuosmanen P, Linder M $\mathrm{B}$, et al. Adhesion and tribological properties of hydrophobin proteins in aqueous lubrication on stainless steel surfaces. RSC Adv 2(26): 9867-9872 (2012)

[39] Escudeiro A, Polcar T, Cavaleiro A. Adsorption of bovine serum albumin on $\mathrm{Zr}$ co-sputtered a-C(: $\mathrm{H})$ films: Implication on wear behaviour. $J$ Mech Behav Biomed Mater 39: 316-327 (2014)

[40] Li X, Guo F, Wong P L. Effect of bovine serum albumin aqueous solution on steel slider surface under hydrodynamic lubrication. Tribol Int 93: 495-501 (2016)

[41] Liu H C, Guo F, Wong P L, Li X. Investigation of adsorbed protein and passive films on hydrodynamic lubricated steel slider surface. Tribol Int 109: 133-139 (2017)

[42] Murakami D, Mawatari N, Sonoda T, Kashiwazaki A, Tanaka M. Effect of the molecular weight of Poly (2-methoxyethyl acrylate) on interfacial structure and blood compatibility. Langmuir 35(7): 2808-2813 (2019)

[43] Raj A, Wang M, Liu C, Ali L, Karlsson N G, Claesson P M, Dédinaite A. Molecular synergy in biolubrication: The role of cartilage oligomeric matrix protein (COMP) in surface-structuring of lubricin. J Colloid Interface Sci 495: 200-206 (2017)

[44] Ghosh S, Choudhury D, Pingguan-Murphy B. Lubricating ability of albumin and globulin on artificial joint implants: A tribological perspective. Int J Surf Sci Eng 10(2): 193-206 (2016)

[45] Nečas D, Vrbka M, Urban F, Křupka I, Hartl M. The effect of lubricant constituents on lubrication mechanisms in hip joint replacements. J Mech Behav Biomed Mater 55: 295-307 (2016)

[46] Su C Y, Chen C C, Huang Y L, Chen S W, Fang H W. Optimization of biomolecular additives for a reduction of friction in the artificial joint system. Tribol Int 111: 220-225 (2017)

[47] Huang R L, Liu X, Ye H J, Su R X, Qi W, Wang L B, He $\mathrm{Z}$ M. Conjugation of hyaluronic acid onto surfaces via the interfacial polymerization of dopamine to prevent protein adsorption. Langmuir 31(44): 12061-12070 (2015)
[48] Almalik A, Benabdelkamel H, Masood A, Alanazi I O, Alradwan I, Majrashi M A, Alfadda A A, Alghamdi W M, Alrabiah H, Tirelli N, et al. Hyaluronic acid coated chitosan nanoparticles reduced the immunogenicity of the formed protein corona. Sci Rep 7(1): 10542 (2017)

[49] Patil N J, Rishikesan S, Nikogeorgos N, Guzzi R, Lee S, Zappone B. Complexation and synergistic boundary lubrication of porcine gastric mucin and branched poly (ethyleneimine) in neutral aqueous solution. Soft Matter 13(3): 590-599 (2017)

[50] Dedinaite A, Pettersson T, Mohanty B, Claesson P M. Lubrication by organized soft matter. Soft Matter 6(7): 1520-1526 (2010)

[51] Samaroo K J, Tan M, Putnam D, Bonassar L J. Binding and lubrication of biomimetic boundary lubricants on articular cartilage. J Orthopaed Res 35(3): 548-557 (2017)

[52] Zhang J, Meng Y G, Tian Y, Zhang X J. Effect of concentration and addition of ions on the adsorption of sodium dodecyl sulfate on stainless steel surface in aqueous solutions. Coll Surf A-Physicochem Eng Aspects 484: 408-415 (2015)

[53] Vogt B D, Lin E K, Wu W L, White C C. Effect of film thickness on the validity of the Sauerbrey equation for hydrated polyelectrolyte films. J Phys Chem B 108(34): 12685-12690 (2004)

[54] Ding S, Zhang J, Tian Y, Huang B L, Yuan Y, Liu C S. Magnesium modification up-regulates the bioactivity of bone morphogenetic protein-2 upon calcium phosphate cement via enhanced BMP receptor recognition and Smad signaling pathway. Coll Surf B: Biointerf 145: 140-151 (2016)

[55] Seror J, Merkher Y, Kampf N, Collinson L, Day A J, Maroudas A, Klein J. Articular cartilage proteoglycans As boundary lubricants: Structure and frictional interaction of surface-attached Hyaluronan and Hyaluronan-Aggrecan complexes. Biomacromolecules 12(10): 3432-3443 (2011)

[56] Seror J, Merkher Y, Kampf N, Collinson L, Day A J, Maroudas A, Klein J. Normal and shear interactions between Hyaluronan-Aggrecan complexes mimicking possible boundary lubricants in articular cartilage in synovial joints. Biomacromolecules 13(11): 3823-3832 (2012) 


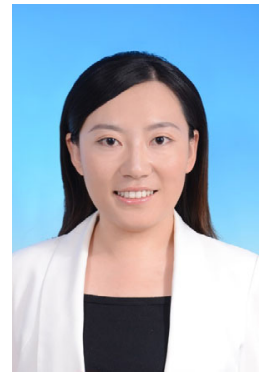

Caixia ZHANG. She received her Ph.D. degree in mechanical engineering in 2015 from Tsinghua University, Beijing, China. After then, she joined the Beijing Key

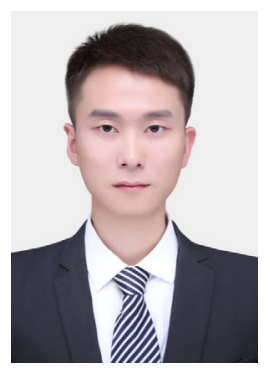

Junmin CHEN. He received his bachelor degree from Luoyang Institute of Science and Technology, China, in 2019. He is currently a graduate student at the Institute of Advanced Manufacturing and

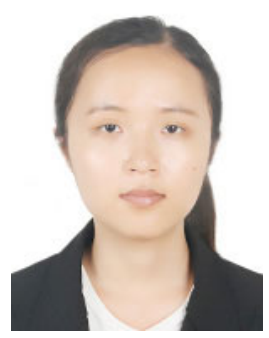

Mengmeng LIU. She received her bachelor degree from Applied Technology College of Soochow University, China. She is currently a graduate student at the Beijing

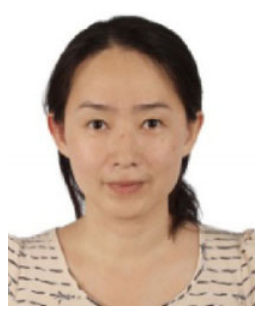

Yuhong LIU. She received her Ph. D. degree in CAS Key Laboratory of Molecular Nanostructure \& Nanotechnology Institute of Chemistry, Chinese Academy of Sciences, Beijing, China in 2005. She joined the State Key Laboratory

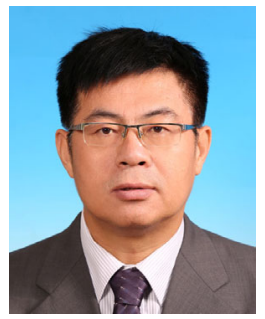

Zhifeng LIU. He received his Ph.D. degree in mechanical engineering from Northeastern University, Shenyang, China. He is the team leader of the Institute
Laboratory of Advanced Manufacturing Technology, Beijing University of Technology. Her research interests include biotribology, superlubricity, and surface and interface analysis.

Intelligent Technology, Beijing University of Technology, China. His research interests include biotribology, superlubricity, surface and interface analysis, and triboelectric nanogenerator.

University of Technology, China. Her research interests include superlubricity and the control of the smart surface and interface.

of Tribology at Tsinghua University in 2005. Her current position is as an associate professor. Her research areas cover nanotribology, nanostructure \& nanotechnology of surface and interface, chemicalmechanical planarization, and water-based lubrication.

of Advanced Manufacturing and Intelligent Technology, Beijing University of Technology. His research interests include heavy-duty machine tools, superlubricity, robot, and assembly technology. 


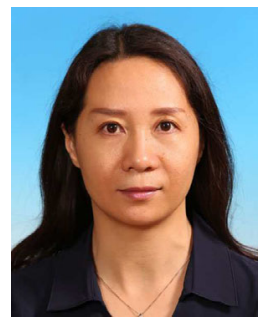

Hongyan CHU. She received her Ph.D. degree in mechanical design and theory in 2003 from the Beijing University of Technology. And now she works at the Institute of Advanced Manufacturing and

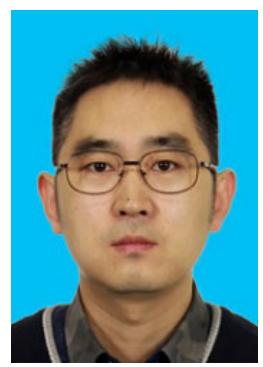

Qiang CHENG. He is a professor of the Institute of Advanced Manufacturing and Intelligent Technology, Beijing University of Technology. He received his Ph.D. degree in mechanical engineering

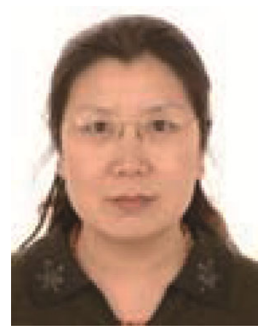

Jianhua WANG. She graduated from Tsinghua University, Beijing, China. After then, she joined the Beijing University of Technology.
Intelligent Technology, Beijing University of Technology. Her research interests include intelligent manufacturing technology, surface and friction characteristics, and contact dynamics of viscoelastic material.

in 2009 from Huazhong University of Science and Technology, Wuhan, China. His main research interests include wear modeling and validation, interface engineering, and precision retaining ability design.

Her research interests include precision machine tools, smart surface, and interface. 